\title{
Hereditary spherocytosis in sisters unmasked by parvovirus infection
}

\author{
N.J. McLellan and N. Rutter \\ University Department of Child Health, Queen's Medical Centre, University Hospital, Nottingham, UK.
}

\begin{abstract}
Summary: Teenage sisters were found to have hereditary spherocytosis when they presented in aplastic crisis associated with otherwise asymptomatic parvovirus infection. Such patients may present a source of cross-infection to others at risk.
\end{abstract}

\section{Introduction}

The cause of the acute marrow aplasia occasionally complicating hereditary haemolytic anaemias was a mystery until 1981 when human parvovirus (HPV) was first associated with transient but intense erythroblastopenia in patients with sickle cell anaemia. ${ }^{1}$ Subsequent reports have documented the same association of HPV infection and aplastic crisis in other hereditary haemolytic anaemias ${ }^{2}$ and the virus may even induce temporary aplasia in the course of autoimmune haemolysis. ${ }^{3}$ Normal children may develop erythema infectiosum ('fifth disease', 'slapped cheek disease') and adults a mild influenza-like illness when infected acutely with HPV. These normal patients are protected from symptoms of marrow suppression by their normal peripheral red cell reserve. ${ }^{2}$ When a haemolytic anaemia first presents in aplastic crisis, diagnosis may be difficult. Summerfield \& Wyatt ${ }^{4}$ have briefly reported this occurrence in a febrile 7 year old with hereditary spherocytosis (HS) and concurrent HPV infection. We now describe two sisters presenting with sudden anaemia in adolescence whose previously unrecognized HS was unmasked by HPV infection.

\section{Case reports}

S.A. was well until January 1985 when she presented aged 13 years with a week's progressive tiredness, dizziness and pallor. She had had no previous episodes of anaemia or jaundice. The only positive findings were striking pallor and $2 \mathrm{~cm}$ splenomegaly.

Investigations revealed: haemoglobin $4.8 \mathrm{~g} / \mathrm{dl}$,

Correspondence: N.J. McLellan, M.R.C.P., Department of Paediatrics, Selly Oak Hospital, Raddlebarn Road, Birmingham B29 6JD, UK.

Accepted: 4 August 1986 platelets $220 \times 10^{9} / 1$, total white cell count $10.6 \times 10^{9} / 1$ (neutrophils $53 \%$, lymphocytes $18 \%$ ), nucleated red cells $4.5 \times 10^{9} / 1$ and large numbers of spherocytes on the peripheral blood film. The serum bilirubin, red cell and serum folates were normal. A direct Coombs' test was negative.

Her mother had had a splenectomy at the age of 11 years, but she was unaware of the reason for this. Review of her notes from 1954 confirmed underlying spherocytosis and indicated that her own father and grandmother had been mildly affected. When S.A.'s two sibs were investigated her brother was normal, but her 15 year old sister, J.A., had spherocytosis (haemoglobin $11.0 \mathrm{~g} / \mathrm{dl}$, reticulocytes $5.8 \%$, increased osmotic fragility with $50 \%$ lysis in $0.52 \%$ saline and numerous spherocytes on the blood film). Her father had a normal blood count. A diagnosis of HS was therefore made in S.A.'s case with evidence of spontaneous recovery from an aplastic crisis. S. A. was treated with a blood transfusion and folic acid supplements and immunized with polyvalent pneumococcal vaccine before uneventful splenectomy 4 months later.

J.A. presented 36 days after her sister with an identical history of 5 days duration. Pallor was the only clinical finding. She had been taking folic acid $5 \mathrm{mg} /$ day for the previous 3 weeks. Blood count showed haemoglobin $6.5 \mathrm{~g} / \mathrm{dl}$, white cell count $7.8 \times 10^{9} / 1$ (neutrophils $51 \%$, lymphocytes $35 \%$ ), platelets $201 \times 10^{9} / 1$, reticulocytes $2.0 \%$ and numerous spherocytes were present on the blood film. She was managed identically to her sister and also recovered uneventfully from later splenectomy.

S.A. and J.A. showed positive IgM titres to HPV when tested 2 weeks after their respective presentations. Titres to a panel of other viruses and mycoplasma remained negative. All other family members had remained well and there had been no known 
symptomatic contacts. Their mother's blood film did not suggest any marrow suppression at, and 2 weeks after, S.A.'s presentation.

\section{Discussion}

This family's spherocytosis was of a relatively mild type as judged by the age of presentation in those affected but HPV infection was sufficient to induce an aplastic crisis and unmask the underlying defect. An underlying congenital haemolytic anaemia should be considered in children presenting for the first time with anaemia and erythroblastopenia.

Little is known of the epidemiology of HPV infection except that the prevalence of HPV antibodies in older children and adults is $30-60 \%{ }^{2,5}$ Systemic HPV infection has recently been induced by nasal inoculation of virus particles in seronegative normal adults. ${ }^{5}$ After an incubation period of about 7 days, intense viraemia occurs for a further week and virus is detected in respiratory secretions. The viraemic phase is associated initially with a mild febrile reaction and then by reticulocytopenia. Later a small reduction in haemoglobin concentration occurs reaching a nadir at 14 days after inoculation. After a further 3 to 4 days, a second phase of illness appears with a rash and arthralgia typical of adult erythema infectiosum. IgMspecific antibodies appear from 9 days after inoculation and an IgG response occurs after the second week.

\section{References}

1. Serjeant, G.R., Mason, K., Topley, J.M. \& Serjeant, B.E. Outbreak of aplastic crisis in sickle cell anaemia associated with parvovirus like agent. Lancet 1981, ii: 595-597.

2. Mortimer, P.P. The 80th year of fifth disease. Br Med J 1984, 289: 338-339.

3. Bertrand, Y., Lefrere, J.J., Leverger, G. et al. Autoimmune haemolytic anaemia revealed by human parvovirus linked erythroblastopaenia (letter). Lancet 1985, ii: 382383.

4. Summerfield, G.P. \& Wyatt, G.P. Human parvovirus infection revealing hereditary spherocytosis (letter). Lancet 1985 , ii: 1070 .

5. Andersen, M.J., Higgins, P.G. \& Davis, R. Experimental
The observation that the viraemia and reticulocytopenia have passed before the rash appears in HPV infection makes sense of earlier findings. Kelleher and colleagues, ${ }^{6}$ for example, detected HPV particles in the serum of a child with known spherocytosis in acute aplastic crisis but only serological evidence of HPV infection could be found in erythema infectiosum.

In the present report, 31 days elapsed between the presentation of the first sister and onset of symptoms in the second. Each could have been infected from outside contacts but there was no known local outbreak of illness. Alternatively, if the first sister had infected the second, then either the incubation period can be considerably longer than 7 days or low grade infectivity persists beyond the duration of aplasia and the second sister was infected closer to the time she became symptomatic.

An episode of possible HPV hospital cross-infection recently prompted Evans and co-workers ${ }^{8}$ to suggest that passive immunization might be appropriate in atrisk contacts of patients with HPV-induced marrow aplasia. However, until the epidemiology is more clearly established, we think it would be sufficient simply to nurse children with congenital haemolytic anaemias in aplastic crisis in a sideroom if contact with other at-risk children is likely. Parents could be advised that there is a chance of at-risk sibs being similarly affected over the next month and that a blook count should be performed if symptoms occur.

parvoviral infection in humans. $J$ Infect Dis 1985,152 257-265.

6. Kelleher, J.F., Luban, N.L.C., Mortimer, P.P. \& Kamimura, T. Human serum parvovirus: a specific cause of aplastic crisis in children with hereditary spherocytosis. J Paediatr 1983, 102: 720-722.

7. Okabe, N., Koboyashi, I., Tatsuzawa, O. \& Mortimer, P.P. Detection of antibodies to human parvovirus in erythema infectiosum (fifth disease). Arch Dis Child 1984, 59: 1016-1019.

8. Evans, J.P., Rossiter, M.A., Kumaran, T.O., Marsh, G.W. \& Mortimer, P.P. Human parvovirus aplasia. Case due to cross infection in a ward. $\mathrm{Br}$ Med $J$ 1984, 288: 681. 